\title{
The Mitochondrial tRNA ${ }^{\text {Ala }}$ T5655C Mutation May Modulate the Phenotypic Expression of tRNA ${ }^{\text {Met }}$ and tRNA ${ }^{\text {Gln }}$ A4401G Mutation in a Han Chinese Family With Essential Hypertension
}

\author{
Yunhong Xu, ${ }^{1} \mathrm{MD}$, Ximing Chen, ${ }^{1} \mathrm{MD}$, Huanliang HuAng, ${ }^{2} \mathrm{MD}$, \\ and Wanting LiU, ${ }^{3} \mathrm{MD}$
}

\begin{abstract}
SUMMARY
Mutations in mitochondrial DNA are associated with the pathogenesis of essential hypertension. We report here the clinical, genetic, and molecular characterization of a three-generation Han Chinese family with essential hypertension. Most strikingly, this family exhibited a high penetrance of essential hypertension. Sequence analysis of the mitochondrial genome showed the presence of a homoplasmic T5655C mutation in tRNA ${ }^{\text {Ala }}$, together with the A4401G mutation in the adjacent region between $\mathrm{RNA}^{\mathrm{Met}}$ and $\mathrm{TNA}^{\mathrm{Gln}}$. Notably, the T5655C mutation was localized at the acceptor arm of tRNA $^{\text {Ala }}$, disrupted the high conserved base-pairing (1A-72T), and may impair the tRNA function. Moreover, the A $4401 \mathrm{G}$ mutation was reported to decrease the steady-state level of tRNA ${ }^{\text {Met }}$ and $\mathrm{tRNA}^{\mathrm{Gln}}$, and consequently caused the mitochondrial dysfunction responsible for hypertension. Taken together, the combination of T5655C and A4401G mutations in mitochondrial tRNA genes may account for the high penetrance and expressivity of hypertension in this Chinese family. Thus, our findings may provide new insight into the pathogenesis of this disorder. (Int Heart J 2017; 58: 95-99)
\end{abstract}

Key words: mt-tRNA, Variants, Pedigree, Blood pressure

$\mathrm{E}$ ssential hypertension $(\mathrm{EH})$ is one of the most important modifiable risk factors for cardiovascular disease and renal disease worldwide. $\mathrm{EH}$ is commonly regarded as a multifactorial disease influenced by both genetic and environmental factors. Familial aggregation of high blood pressure, despite different environmental factors, suggests that genetic factors are involved in the etiology of hypertension. ${ }^{1)}$

$\mathrm{EH}$ can be caused by single gene mutations, resulting from interactions between the environment and inherited risk factors. It is now generally believed that human hypertension is a condition associated with endothelial dysfunction and oxidative stress. ${ }^{2)}$ Mitochondrial dysfunction has been potentially implicated in both human and experimental hypertension., Human mitochondrial DNA (mtDNA) is a double-stranded circular molecule with 16,569 bp encoding 37 genes: 13 for essential subunits of the oxidative phosphorylation (OXPHOS) system, 2 for rRNAs, and 22 for tRNAs required for mitochondrial protein synthesis. ${ }^{5)}$ All of these mt-tRNAs form a highly conserved cloverleaf structure, containing the acceptor, anticodon, and T stems, as well as T $\psi \mathrm{C}, \mathrm{D}$, and anticodon loops. Due to the lack of histone protection and a poor DNA repair system, mtDNA has a higher mutation rate than nuclear DNA. Most recently, several mt-tRNA mutations have been reported to be associated with $\mathrm{EH}$; these mutations include the A4435G in the tRNA ${ }^{\text {Met }}$ gene, ${ }^{6}$ A12330G in the tRNA ${ }^{\text {Leu(CUN) }}$ gene $^{7)}$ and
A4295G in the tRNA ${ }^{\text {Ile }}$ gene. ${ }^{8)}$ These mutations can result in translational defects and consequently mitochondrial respiratory chain dysfunction, and are associated with the pathogenesis of $\mathrm{EH}$.

In order to identify novel mtDNA mutations involved in the pathogenesis of $\mathrm{EH}$ in a Chinese population, we initiated a systematic and extended mutational screening of mtDNA in a large cohort of EH subjects from Guangzhou area, Guangdong Province. Here we describe a Han Chinese family with EH carrying the homoplasmic A4401G and T5655C mutations in the mitochondrial genome.

\section{MeTHODS}

Subjects: As part of a genetic screening program for $\mathrm{EH}$, a Chinese Han family with maternally inherited EH was identified at the Third Affiliated Hospital of Guangzhou Medical University. Informed consent, blood samples, and clinical evaluation were obtained from all participating family members under protocols approved by the Ethics Committee of Guangzhou Medical University. Members of this family were interviewed and evaluated to identify both personal and medical histories of EH and other clinical abnormalities. A healthy control population of 200 individuals was obtained from a

From the Departments of ${ }^{1}$ Cardiology, ${ }^{2}$ Emergency, and ${ }^{3}$ Physical Examination Center, The Third Affiliated Hospital of Guangzhou Medical University, Guangzhou, China.

Address for correspondence: Ximing Chen, MD, Department of Cardiology, the Third Affiliated Hospital of Guangzhou Medical University, No. 63, Duobao Road, Liwan District, Guangzhou, 510150, P.R. China. E-mail: chenxmgz@163.com

Received for publication April 28, 2016. Revised and accepted June 20, 2016.

Released in advance online on J-STAGE January 20, 2017.

All rights reserved by the International Heart Journal Association. 
panel of unaffected Han Chinese residents from the same area. Members of this Chinese family underwent a physical examination, laboratory assessment of cardiovascular disease risk factors, and routine electrocardiography. An experienced physician measured the systolic and diastolic blood pressures of subjects with a mercury column sphygmomanometer using a standard protocol. The first and fifth Korotkoff sounds were taken to be indicative of systolic and diastolic blood pressures, respectively. The average of 3 such readings was taken as the examination blood pressure. EH was defined according to the recommendation of the Joint National Committee on Detection, Evaluation and Treatment of High Blood Pressure (JNC VI) as a systolic blood pressure of $140 \mathrm{mmHg}$ or higher and/or a diastolic blood pressure of $90 \mathrm{mmHg}$ or greater. ${ }^{9}$

Mutational analysis of mitochondrial tRNA (mt-tRNA) genes: To determine whether mt-tRNA mutations played important roles in EH, we performed a screening for the candidate pathogenic mt-tRNA mutations in the proband (III-4) and matrilineal relatives (I-2, II-4, II-6, III-3). All these experiments were carried out at the Guangzhou Institute of Obstetrics and Gynecology of the Third Affiliated Hospital of Guangzhou Medical University. Briefly, the genomic DNA was isolated from whole blood cells of participants using Puregene DNA Isolation Kits (Gentra Systems, Minneapolis, MN). The entire mt-tRNA genes of the participants were PCR amplified using the primers as previously described. ${ }^{10)}$ Each fragment was purified and subsequently analyzed by direct sequencing in an ABI 3700 automated DNA sequencer (Applied Biosystems, Inc, Foster City, CA) using a Big Dye Terminator Cycle sequencing reaction kit. The resultant sequence data were compared with the reversed Cambridge sequence (GenBank accession No. NC_012920). ${ }^{11)}$

Phylogenetic conservation analysis: To analyze the phylogeny of mt-tRNA mutations, we used vertebrate mtDNA sequences for inter-specific analysis; these species included Elephas maximus, Macropus robustus, Homo sapiens, Hylobates lar, Macaca mulatta, Pan paniscus, Pan troglodytes, Capra hircus, Lama pacos, and Orycteropus afer. The conservation index (CI) was then calculated by comparing the human nucleotide variants with the other species. Notably, the $\mathrm{CI} \geq 75 \%$ was considered as functional potential. ${ }^{12)}$

Determination of pathogenicity: We further used the pathogenicity scoring system to predict the potential pathogenic role of mt-tRNA mutations. According to that standard, an mttRNA mutation was regarded as "definitely pathogenic" with a total score of more than 11 points, if the score was between 7-10 points, it belonged to "possibly pathogenic", whereas a score that was less than 6 points should be classified as "neutral polymorphism". ${ }^{13,14)}$

\section{RESULTS}

Clinical features: The proband (III-4) was a 32-year-old woman who came from Guangzhou area of Guangdong Province. She first experienced hypertension when she was 29 years-old. She went to the hypertension clinic of the Third Affiliated Hospital of Guangzhou Medical University for further clinical evaluations, at which time her blood pressure was $155 / 90 \mathrm{mmHg}$. Physical examination, laboratory assessment of cardiovascular risk factors, and routine electrocardiography showed that she did not have any other clinical abnormalities such as diabetes, vision loss, hearing impairment, or renal and neurological disorders. Therefore, she exhibited a typical EH. As shown in Figure 1 and Table I, the average age at onset of $\mathrm{EH}$ in the maternal kindred varied from 29 years to 70 years, with an average of 47 years. There was no evidence that any member of this family had any other cause to account for EH. Therefore, the inheritance pattern of this family was consistent with maternal inheritance.

Mutational analysis of the mt-tRNA genes: Because previous studies showed that mt-tRNAs were the hot spots for pathogenic mutations associated with $\mathrm{EH},{ }^{15)}$ we aimed to evaluate the role of mt-tRNA mutations in EH expression. As shown in Figure 2, the homoplasmic A4401G and T5655C mutations were identified by direct Sanger sequencing. Of note, the wellknown A4401G mutation was localized at the adjacent site of tRNA $^{\text {Met }}$ and tRNA ${ }^{\text {Gln }}$. In contrast, the T5655C mutation occurred at the 5' end of tRNA ${ }^{\text {Ala }}$ and the first nucleotide adenine was replaced with guanine (Figure 4). Moreover, as shown in Figure 3, the T5655C mutation was highly conserved between different species $(\mathrm{CI}=100 \%)$, which indicated that it may play an important role in EH expression (Figure 3).

Determination of pathogenicity: According to the revised pathogenicity scoring system, we classified the tRNA ${ }^{\text {Ala }}$ T5655C mutation as "definitely pathogenic" with a total score of 17 points (Table II).

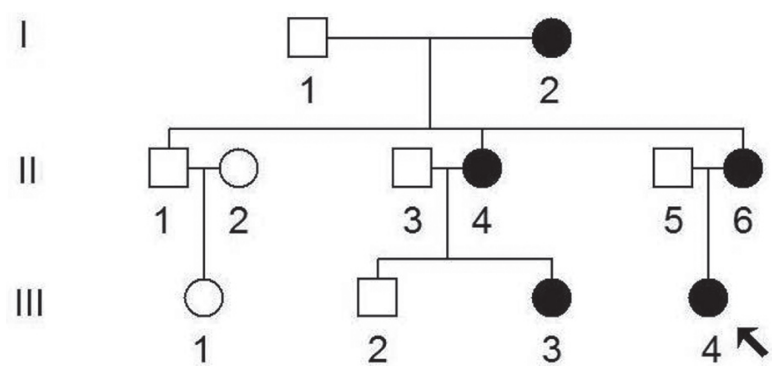

Figure 1. A Han Chinese family with essential hypertension (EH). Affected individuals are indicated by filled symbols, and the arrow denotes the proband (III-4).

Table I. Summary of Clinical Data for Several Members in This Chinese Family

\begin{tabular}{|c|c|c|c|c|c|c|}
\hline Subject & Gender & Age at test & Age at onset & $\begin{array}{c}\text { Systolic Pressure } \\
(\mathrm{mmHg})\end{array}$ & $\begin{array}{c}\text { Diastolic Pressure } \\
(\mathrm{mmHg})\end{array}$ & Presence of mt-tRNA mutations \\
\hline $\mathrm{I}-2$ & Female & 84 & 70 & 145 & 95 & T5655C, A4401G \\
\hline II-4 & Female & 59 & 55 & 160 & 80 & T5655C, A4401G \\
\hline II-6 & Female & 58 & 54 & 180 & 95 & $\mathrm{~T} 5655 \mathrm{C}, \mathrm{A} 4401 \mathrm{G}$ \\
\hline III-3 & Female & 33 & 30 & 145 & 90 & T5655C, A4401G \\
\hline III-4 & Female & 32 & 29 & 155 & 90 & T5655C, A4401G \\
\hline III-1 & Male & 30 & I & 130 & 75 & None \\
\hline
\end{tabular}




\section{DiSCUSSION}

In this study, we have performed the clinical, genetic, and molecular characterization of a Han Chinese family with high penetrance of $\mathrm{EH}$. EH as the sole clinical phenotype only presented in the maternal lineage of this pedigree, the inherited pattern provided a clear indication for the mtDNA mutations being responsible for the phenotype. In particular, the age of onset of this family ranged from 29 to 70 years, and the matrilineal relatives had an earlier age at onset of $\mathrm{EH}$, suggesting that mitochondrial sequence variants may be a risk factor for this disease.

Mitochondria are important vital energy producing organelles in eukaryotic cells and are primarily responsible for generating ATP by OXPHOS. ${ }^{16)}$ Recently, increasing evidence has shown that mitochondrial dysfunction caused by mtDNA mutations plays an important role in the development of $\mathrm{EH}$, and mitochondrial dysfunction will increase the production of reactive oxygen species (ROS), which will lead to oxidative stress, loss of nitric oxide signaling and endothelial barrier function, and infiltration of leukocytes into the vascular wall, and thus, contribute to high blood pressure. ${ }^{17,18)}$ Today, approximately 200 pathogenic mutations had been mapped to mttRNA genes (http://www.mitomap.org/MITOMAP), ${ }^{19)}$ empha- sizing the importance of mt-tRNAs for mitochondrial function. In this study, we identified two mutations: $A 4401 G$ in the junction between the tRNA ${ }^{\text {Met }}$ at the heavy strand (H-strand) and $\mathrm{tRNA}^{\mathrm{Gln}}$ at the light strand (L-strand), ${ }^{20)}$ as well as the T5655C in tRNA ${ }^{\text {Ala }}$ (Figure 2 and 4). These mutations were present only in matrilineal relatives of this family in the homoplasmic form but not in 200 healthy controls. Of these, the

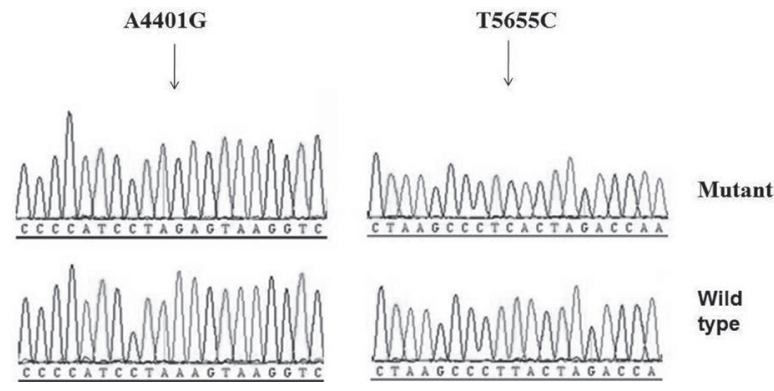

Figure 2. Identification of mt-tRNA mutations in the positions: 4401 in tRNA $^{\text {Met }}$ and tRNA ${ }^{\text {Gln }}, 5655$ in the tRNA ${ }^{\text {Ala }}$. Partial sequence chromatograms of the fragments spanning the corresponding regions of mt-tRNAs from the proband (III-4) and the healthy control. Arrows indicate the location of the base changes.

\begin{tabular}{|c|c|c|c|c|c|c|c|c|c|c|c|c|c|c|c|}
\hline \multirow[t]{2}{*}{ Qrganism } & \multicolumn{2}{|l|}{ Acc-stem } & \multirow{2}{*}{$\begin{array}{l}\text { Destem } \\
10\end{array}$} & \multirow[t]{2}{*}{ D. loop } & \multicolumn{2}{|l|}{ D.stern } & $\begin{array}{l}\text { Atco } \\
\text { Stem }\end{array}$ & $\begin{array}{l}\text { Antited } \\
\text { looip }\end{array}$ & \multirow{2}{*}{ Acostem } & $\begin{array}{c}\text { Vo } \\
\text { region }\end{array}$ & T-stem & \multirow[t]{2}{*}{ Tloop } & \multirow{2}{*}{$\begin{array}{l}\text { T-stem } \\
61\end{array}$} & \multicolumn{2}{|l|}{ ACcstem } \\
\hline & 1 & 8 & & & 22 & & 27 & 32 & & 44 & 49 & & & 66 & 73 \\
\hline Elephas maximus & $\triangle A G G G C T$ & TA & GTTT & AGTTA & $A A G C$ & $G$ & TCTGA & TTTGCAT & TCAGT & TGAT & GTAAG & GTGATAT & CTTGC & AGTCCTT & A \\
\hline Macropus robustus & AAGAATT & TA & GCTT & AATGA & AAGT & $G$ & TTCGA & TTTGCGT & TCGGA & TGAT & GCAAG & ATAAAGG & CTTGT & AATTCTT & A \\
\hline Homo sapiens & AAGGGCT & TA & GCTT & AATTA & AAGT & $G$ & GCTGA & TTTGCGT & TCAGT & TGAT & GCAGA & GTGGGGT & TTTGC & AGTCCTT & A \\
\hline Hylobates lar & AAGGGCT. & TA & GCTT & AATTA & AAGT & $G$ & ACTGA & TTTGCGT & TCGGT & TGAT & GCAAA & GTGGGC & TTTGC & AGTCCTT & G \\
\hline Macaca mulatta & $A A G G G C T$ & TA & GCTT & AATTA & AAGT & $G$ & GTTGA & TTTGCGT & TCAAT & TGAT & GCAGA & GTAGGTG & TTTGC & AGTCCTT & A \\
\hline Pan paniscus & AAGGGCT & TA & GCTT & AATTA & AAGT & $G$ & GCTGA & TTTGCGT & TCAGT & TGAT & GCAGA & GTGGGGT & TTTGC & AGTCCTT & A \\
\hline Pan troglodytes & $\triangle A G G G C T$ & TA & GCTT & AATTA & AAGT & $G$ & GCTGA & TTTGCGT & TCAGT & TGAT & GCAGA & GTGGGGT & TTTGC & AGTCCTT & A \\
\hline Capra hircus & AAGGATT & TA & GCTT & AATTA & AAGT & G & GTTGA & TTTGCAT & TCAAT & TGAT & GTAAG & ATATGGT & CTTGC & AGTCCTT & A \\
\hline Lama pacos & $\triangle A G G G C T$ & TA & GCTT & AATTA & AAGT & A & GTTGA & TTTGCAT & TCAAT & TGAT & GTAGG & ATAGAGT & CTTCC & AGTCCTT & A \\
\hline Orycteropus afer & AAGGCCT & TA & GCTT & AATTA & AAGT & A & ACTGA & TTTGCGT & TCAGT & GGAT & GTAGG & ATAGAGT & CTTCC & AGTCCTT & A \\
\hline
\end{tabular}

Figure 3. Alignment of tRNA ${ }^{\text {Ala }}$ gene from different species. Arrow indicates the position 1, corresponding to the T5655C mutation.

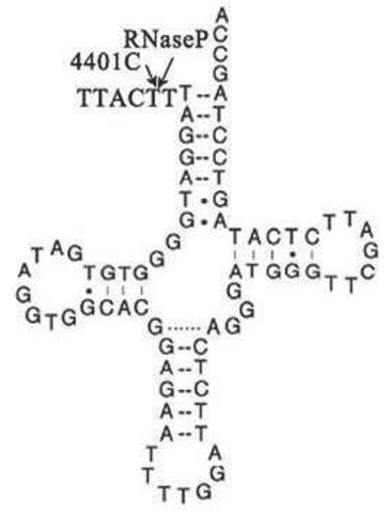

tRNA $^{\mathrm{Gln}}$

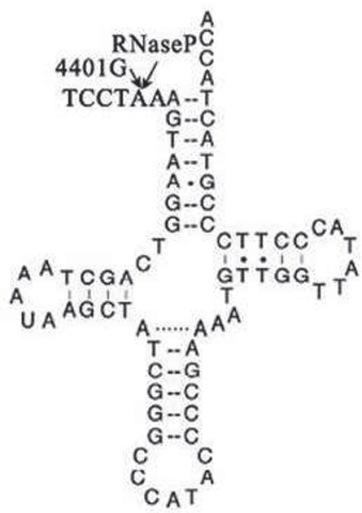

tRNA ${ }^{\text {Met }}$

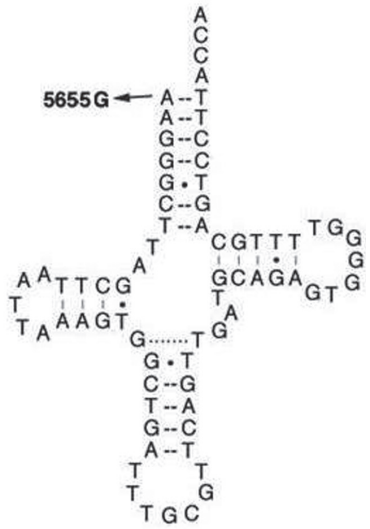

tRNA ${ }^{\mathrm{Ala}}$

Figure 4. Location of the A4401G mutation in the junction of tRNA ${ }^{\mathrm{Met}}$ and $\mathrm{TRNA}^{\mathrm{Gln}}$, and the T5655C mutation in the tRNA $^{\text {Ala }}$. Cloverleaf structures of human mt-tRNAs are derived from the Mitomap database (http://www.mitomap.org/ MITOMAP). The arrows indicate the positions of these mtDNA mutations. 
Table II. Pathogenicity Scoring System for T5655C Mutation

\begin{tabular}{|c|c|c|c|}
\hline Scoring criteria & T5655C mutation & Score & Classification \\
\hline More than one independent report & Yes & 2 & $\leq 6$ points: neutral polymorphisms; \\
\hline Evolutionary conservation of the base pair & No changes & 2 & \\
\hline Variant heteroplasmy & No & 0 & 7-10 points: possibly pathogenic; \\
\hline Segregation of the mutation with disease & Yes & 2 & $11-13$ points (not including evidence from single \\
\hline Histochemical evidence of mitochondrial disease & Strong evidence & 2 & \\
\hline Biochemical defect in complex I, III or IV & Yes & 2 & fiber, steady-state level): probably pathogenic; \\
\hline $\begin{array}{l}\text { Evidence of mutation segregation with biochemical defect from } \\
\text { single-fiber studies }\end{array}$ & Yes & 2 & $\geq 11$ points (including trans-mitochondrial cybrid \\
\hline $\begin{array}{l}\text { Mutant mt-tRNA steady-state level or evidence of pathogenicity in } \\
\text { trans-mitochondrial cybrid studies }\end{array}$ & Strong evidence & 5 & studies): definitely pathogenic. \\
\hline Maximum score & & 17 & Definitely pathogenic \\
\hline
\end{tabular}

A4401G mutation was identified in several Chinese families with $\mathrm{EH}$ and was implicated to cause a failure in tRNA metabolism. ${ }^{21,22)}$ The 5 ' end of the flanking sequence is 4401A/AGTAAG in the tRNA ${ }^{\text {Met }}$ gene, whereas the 5'end of the flanking sequence is $4401 \mathrm{~T} / \mathrm{TGAGAT}$ in the tRNA ${ }^{\text {Gln }}$ gene. ${ }^{23)}$ In fact, the processing of mt-tRNAs requires the precise endonucleolytic cleavage at both the 3' and 5' ends catalyzed by RNase P and 3' endonuclease. ${ }^{24,25)}$ Thus, the A4401G mutation may affect the reaction efficiency of the RNase P involved in tRNA ${ }^{\mathrm{Met}}$ and $\mathrm{RNA}^{\mathrm{Gln}} 5$ ' end metabolism. Functional characterization of cell lines carrying this mutation showed a significant reduction of the steady-state level of tRNA ${ }^{\text {Met }}$ and tRNA ${ }^{\text {Gln }}$. In addition, the $\mathrm{A} 4401 \mathrm{G}$ mutation caused the defects in mitochondrial protein synthesis and respiratory chain function. On the other hand, the homoplasmic T5655C mutation, combined with the ND1 T3308C mutation, was first described in an African family with maternally inherited aminoglycoside-induced and nonsyndomic hearing impairment carrying the tRNA ${ }^{\mathrm{Ser}(\mathrm{UCN})}$ T7511C mutation. ${ }^{26)}$ As shown in Figure 3, the T5655C mutation occurred at the position which was highly conserved between different species. Interestingly, the T5655C mutation produced an approximate $50 \%$ reduction in the tRNA ${ }^{\text {Ala }}$ level, whereas the ND1 T3308C mutation caused significant decreases both in the amount of ND1 mRNA and co-transcribed tRNA $^{\text {Leu(UUR) }}$ in mutant cells. Therefore, the co-existence of the T5655C mutation and T3308C mutation may contribute to the high penetrance and expressivity of deafness-associated T7511C mutation. ${ }^{27)}$ Moreover, a previous study indicated that the $\mathrm{T} 5655 \mathrm{C}$ mutation played an important role in the recognition of aminoacyl-tRNA synthetase. ${ }^{23)}$ The significant decrease in $\mathrm{RRNA}^{\text {Ala }}$ steady-state levels in the mutant cybrid carrying the T5655C mutation may result from a failure to aminoacylate properly and of post-transcriptional modification of this tRNA. $^{27)}$

Based on these findings, we propose that the molecular mechanism underlying the T5655C and A4401G mutations in the pathogenesis of EH may be as follows: first, these mutations caused the alternation of mt-tRNA secondary structure which will subsequently result in the failure in mt-tRNA metabolism, including decreases in the tRNA steady state level and aminoacylation, which will eventually lead to decreased mitochondrial protein synthesis. Defects in mitochondrial translation consequently lead to a respiratory phenotype and a decline in ATP production below the threshold level required for endothelial cell function, ${ }^{28)}$ thereby contributing to the high blood pressure. This is the first time that a study has provided direct evidence for the hypertension associated mitochondrial
T5655C and A4401G mutations.

\section{Disclosure}

The authors have no conflicts of interest to disclose.

\section{REFERENCES}

1. Nakanishi T. Pulmonary arterial hypertension associated with congenital heart disease. Personal perspectives. Int Heart J 2015; 56: S1-3. (Review)

2. Redón J, Oliva MR, Tormos C, et al. Antioxidant activities and oxidative stress byproducts in human hypertension. Hypertension 2003; 41: 1096-101.

3. Arrell DK, Elliott ST, Kane LA, et al. Proteomic analysis of pharmacological preconditioning: novel protein targets converge to mitochondrial metabolism pathways. Circ Res 2006; 99: 706-14.

4. Yagi H, Sumino H, Yoshida K, et al. Biological antioxidant potential negatively correlates with carotid artery intima-media thickness. Int Heart J 2016; 57: 220-5.

5. Chomyn A, Attardi G. MtDNA mutations in aging and apoptosis. Biochem Biophys Res Commun 2003; 304: 519-29. (Review)

6. Liu Y, Li R, Li Z, et al. Mitochondrial transfer RNAMet 4435A>G mutation is associated with maternally inherited hypertension in a Chinese pedigree. Hypertension 2009; 53: 1083-90.

7. Teng L, Zheng J, Leng J, DingY. Clinical and molecular characterization of a Han Chinese family with high penetrance of essential hypertension. Mitochondrial DNA 2012; 23: 461-5.

8. Li Z, Liu Y, Yang L, Wang S, Guan MX. Maternally inherited hypertension is associated with the mitochondrial tRNA(Ile) A4295G mutation in a Chinese family. Biochem Biophys Res Commun 2008; 367: 906-11.

9. The sixth report of the Joint National Committee on prevention, detection, evaluation and treatment of high blood pressure. Arch Intern Med 1997; 157: 2413-46.

10. Qin Y, Xue L, Jiang P, et al. Mitochondrial tRNA variants in Chinese subjects with coronary heart disease. J Am Heart Assoc 2014; 3: $\mathrm{e} 000437$.

11. Anderson S, Bankier AT, Barrell BG, et al. Sequence and organization of the human mitochondrial genome. Nature 1981; 290: 457-65.

12. Wang L, Chen ZJ, Zhang YK, et al. The role of mitochondrial tRNA mutations in lung cancer. Int J Clin Exp Med 2015; 8: 13341-6.

13. González-Vioque E, Bornstein B, Gallardo ME, FernándezMoreno MÁ, Garesse R. The pathogenicity scoring system for mitochondrial tRNA mutations revisited. Mol Genet Genomic Med 2014; 2: 107-14.

14. Yarham JW, Al-Dosary M, Blakely EL, et al. A comparative analysis approach to determining the pathogenicity of mitochondrial 
tRNA mutations. Hum Mutat 2011; 32: 1319-25.

15. Ding Y, Xia B, Yu J, Leng J, Huang J. Mitochondrial DNA mutations and essential hypertension. Int J Mol Med 2013; 32: 768-74. (Review)

16. Wallace DC. Bioenergetics and the epigenome: interface between the environment and genes in common diseases. Dev Disabil Res Rev 2010; 16: 114-9.

17. Marian AJ. Mitochondrial genetics and human systemic hypertension. Circ Res 2011; 108: 784-6.

18. Kinugawa S, Takada S, Matsushima S, Okita K, Tsutsui H. Skeletal Muscle Abnormalities in Heart Failure. Int Heart J 2015; 56 : 475-84.

19. Ruiz-Pesini E, Lott MT, Procaccio V, et al. An enhanced MITOMAP with a global mtDNA mutational phylogeny. Nucleic Acids Res 2007; 35: D823-8.

20. Ojala D, Montoya J, Attardi G. tRNA punctuation model of RNA processing in human mitochondria. Nature 1981; 290: 470-4.

21. Zhu HY, Wang SW, Liu L, et al. A mitochondrial mutation A4401G is involved in the pathogenesis of left ventricular hypertrophy in Chinese hypertensives. Eur J Hum Genet 2009; 17: 1728.

22. Li R, Liu Y, Li Z, Yang L, Wang S, Guan MX. Failures in mitochondrial tRNAMet and tRNAGln metabolism caused by the novel $4401 \mathrm{~A}>\mathrm{G}$ mutation are involved in essential hypertension in a
Han Chinese Family. Hypertension 2009; 54: 329-37.

23. Florentz C, Sohm B, Tryoen-Tóth P, Pütz J, Sissler M. Human mitochondrial tRNAs in health and disease. Cell Mol Life Sci 2003; 60: 1356-75. (Review)

24. Holzmann J, Frank P, Löffler E, Bennett KL, Gerner C, Rossmanith W. RNase P without RNA: identification and functional reconstitution of the human mitochondrial tRNA processing enzyme. Cell 2008; 135: 462-74

25. Levinger L, Jacobs O, James M. In vitro 3'-end endonucleolytic processing defect in a human mitochondrial tRNA(Ser(UCN)) precursor with the U7445C substitution, which causes nonsyndromic deafness. Nucleic Acids Res 2001; 29: 4334-40.

26. Sue CM, Tanji K, Hadjigeorgiou G, et al. Maternally inherited hearing loss in a large kindred with a novel T7511C mutation in the mitochondrial DNA tRNA(Ser(UCN)) gene. Neurology 1999; 52: 1905-8.

27. Li X, Fischel-Ghodsian N, Schwartz F, Yan Q, Friedman RA, Guan MX. Biochemical characterization of the mitochondrial tRNASer(UCN) T7511C mutation associated with nonsyndromic deafness. Nucleic Acids Res 2004; 32: 867-77.

28. Yang Q, Kim SK, Sun F, et al. Maternal influence on blood pressure suggests involvement of mitochondrial DNA in the pathogenesis of hypertension: the Framingham Heart Study. J Hypertens 2007; 25: 2067-73 